\title{
Magnetic Interactions in Rare-Earth Metals from Inelastic Neutron Scattering
}

\author{
Bjerrum Møller, Hans; Houmann, Jens Christian Gylden; Mackintosh, A. R.
}

Published in:

Physical Review Letters

Link to article, DOI:

10.1103/PhysRevLett.19.312

Publication date:

1967

Document Version

Publisher's PDF, also known as Version of record

Link back to DTU Orbit

Citation (APA):

Bjerrum Møller, H., Houmann, J. C. G., \& Mackintosh, A. R. (1967). Magnetic Interactions in Rare-Earth Metals from Inelastic Neutron Scattering. Physical Review Letters, 19(6), 312-314.

https://doi.org/10.1103/PhysRevLett.19.312

\section{General rights}

Copyright and moral rights for the publications made accessible in the public portal are retained by the authors and/or other copyright owners and it is a condition of accessing publications that users recognise and abide by the legal requirements associated with these rights.

- Users may download and print one copy of any publication from the public portal for the purpose of private study or research.

- You may not further distribute the material or use it for any profit-making activity or commercial gain

- You may freely distribute the URL identifying the publication in the public portal 
MAGNETIC INTERACTIONS IN RARE-EARTH METALS FROM INELASTIC NEUTRON SCATTERING

\author{
H. Bjerrum Mфller \\ Research Establishment Ris $\varnothing$, Roskilde, Denmark \\ and \\ J. C. Gylden Houmann and A. R. Mackintosh \\ Technical University, Lyngby, Denmark \\ (Received 12 June 1967)
}

We have measured the magnon dispersion relations in the $c$ direction over a wide temperature range in ferromagnetic $\mathrm{Tb}$, and in both the ferromagnetic and spiral phases of a $\mathrm{Tb}-10 \%$ Ho alloy. The indirect exchange interaction deduced from these measurements can be related to the Fermi surface, and its temperature dependence reflects the effect of the magnetization on the conduction-electron energy bands. The exchange splitting of the energy bands is also manifested in the measurements of the magnon lifetimes, which are primarily determined by interaction with the conduction electrons. A quasilocalized resonant magnon mode has been observed in the alloy and a large interaction occurs between magnons and transverse phonons propagating in the $c$ direction, possibly mediated by the presence of this resonance.

The Hamiltonian used to describe the magnetic system is

$$
H=-\sum_{i<j} J\left(\overrightarrow{\mathbf{R}}_{i}-\overrightarrow{\mathbf{R}}_{j}\right) \overrightarrow{\mathbf{S}}_{i} \cdot \overrightarrow{\mathrm{S}}_{j}+\sum_{j}\left\{B S_{z j}{ }^{2}+\frac{1}{2} G\left[\left(S_{x j}+i S_{y j}\right)^{6}+\left(S_{x j}-i S_{y j}\right)^{6}\right]\right\},
$$

where $J\left(\overrightarrow{\mathrm{R}}_{i}-\overrightarrow{\mathrm{R}}_{j}\right)$ is the exchange interaction between ions at $\overrightarrow{\mathrm{R}}_{i}$ and $\overrightarrow{\mathrm{R}}_{j}$, and $B$ and $G$ describe the twofold and sixfold anisotropy, respectively. We have used $\vec{S}$ to denote the total angular momentum operator. This Hamiltonian may be diagonalized to give the magnon energies in the $c$ direction, in the ferromagnetic and spiral phases, respectively, $\operatorname{as}^{1,2}$

$$
\begin{gathered}
\hbar \omega(\overrightarrow{\mathrm{q}})=S\left\{[J(0)-J(\overrightarrow{\mathrm{q}})]^{2}+2\left(B+21 G S^{4}\right)[J(0)-J(\overrightarrow{\mathrm{q}})]+72 G S^{4}\left[B+3 G S^{4}\right]\right\}^{1 / 2}, \\
\hbar \omega(\overrightarrow{\mathrm{q}})=S\left\{\left[J(\overrightarrow{\mathrm{Q}})-\frac{1}{2} J(\overrightarrow{\mathrm{Q}}+\overrightarrow{\mathrm{q}})-\frac{1}{2} J(\overrightarrow{\mathrm{Q}}-\overrightarrow{\mathrm{q}})\right][J(\overrightarrow{\mathrm{Q}})-J(\overrightarrow{\mathrm{q}})+2 B]\right\}^{1 / 2},
\end{gathered}
$$

where

$$
J(\overrightarrow{\mathrm{q}})=\sum_{i} J\left(\overrightarrow{\mathrm{R}}_{i}\right) \exp \left(\overrightarrow{i \mathrm{q}} \cdot \overrightarrow{\mathrm{R}}_{i}\right)
$$

$\vec{Q}$ is the wave vector of the spiral, and we have used the double zone representation. In the spiral phase the sixfold anisotropy has been assumed negligible.

The magnon dispersion relations for $\mathrm{Tb}$ at $90^{\circ} \mathrm{K}$ have been presented previously. ${ }^{3}$ In agreement with Eq. (2), the energy is finite at $\vec{q}=0$, due to the magnetic anisotropy, and rises quadratically at low $\overrightarrow{\mathrm{q}}$. The degeneracy at $K$ is consistent with the symmetry of the spinspace group, ${ }^{4}$ with isotropic exchange, as are more recent measurements. As the temperature is increased, $J(0)-J(\vec{q})$ scales generally as the ordered moment, ${ }^{5}$ in agreement with the prediction of Tyablikov, ${ }^{6}$ although the detailed dependence on $\vec{q}$ also changes somewhat.
The addition of $10 \%$ Ho to $\mathrm{Tb}$ increases the tendency towards spiral ordering, and the spiral structure is stable from 221 to $195^{\circ} \mathrm{K}$. Correspondingly, as shown in Fig. $1(\mathrm{~b}), J(\overrightarrow{\mathrm{q}})$ in the ferromagnetic phase is flatter at small $q$ than it is in pure $\mathrm{Tb}$. From the $\overrightarrow{\mathrm{q}}$ dependence of the neutron group intensities ${ }^{7}$ at $110^{\circ} \mathrm{K}$, we find that $B=0.25 \mathrm{meV} /$ ion and from the magnitude of the energy gap at $\overrightarrow{\mathrm{q}}=0, G S^{4}=8.7 \times 10^{-4}$ $\mathrm{meV} /$ ion. The twofold anisotropy is in fair agreement with the torque measurements of Rhyne and Clark, ${ }^{8}$ but $G$ is much greater than they deduce from magnetostriction measurements. This discrepancy may be partially accounted for by the dependence of the zeropoint magnon energy on magnetization. ${ }^{9}$ In interpreting our results, we have used the anisotropy constants derived from our neutron measurements at $110^{\circ} \mathrm{K}$, but scaled with tem- 

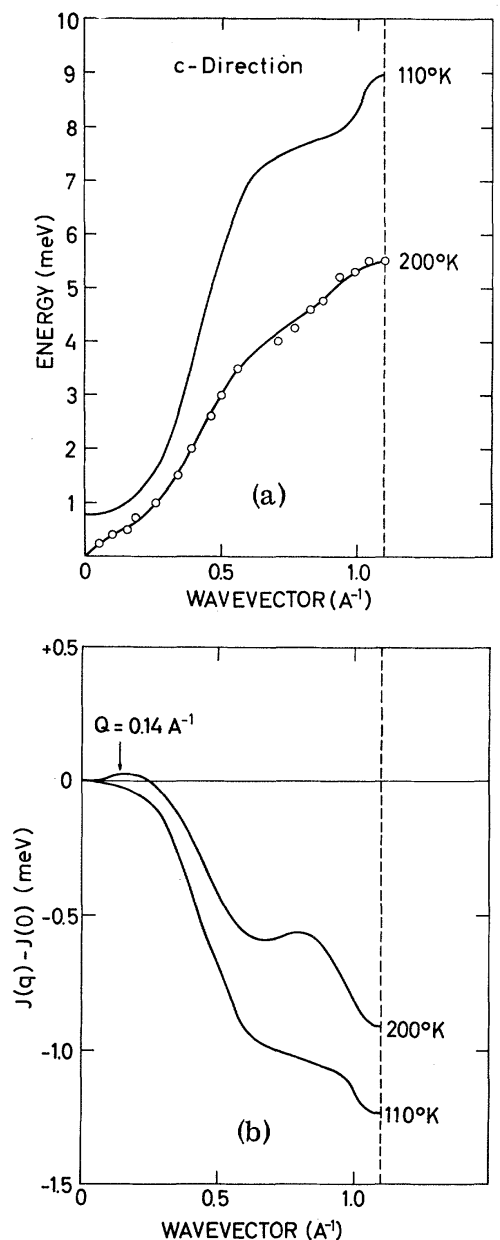

FIG. 1. (a) Magnon dispersion relations in $\mathrm{Tb}-10 \%$ Ho in the ferromagnetic and spiral phases. The full line for the ferromagnetic phase is derived principally from the experimental points of Fig. 2(a), while the form of the magnon dispersion curve in the region of the magnon-phonon interaction is determined from measurements at higher temperatures. The full line for the spiral phase is a weighted least-squares fit of the experimental points to Eq. (3). (b) $J(\vec{q})-J(0)$ deduced from the dispersion curves, using Eqs. (2) and (3). The dashed line is drawn at the position of the (001) reciprocal lattice point.

perature according to the results of Rhyne and Clark. ${ }^{8}$

The magnon dispersion relation in the $c$ direction has also been measured in the spiral phase of the alloy, and the results are shown in Fig. 1(a). In accordance with Eq. (3), the magnon energy rises linearly from 0 and, because of the twofold anisotropy, is finite at $\overrightarrow{\mathrm{q}}=\overrightarrow{\mathrm{Q}}$. The values of $J(\overrightarrow{\mathrm{q}})-J(0)$, deduced from these measurements, are shown in Fig. 1(b). The most striking features are the maxima which occur around $\overrightarrow{\mathbf{Q}}$ and $\vec{\tau}-\vec{Q}$, where $\vec{\tau}$ is the (001) reciprocal lattice point. The former is responsible for the stability of the spiral structure. $J(\vec{q})$ is directly related to the conduction-electron energy-band structure and, in the periodic zone scheme, is given by an expression of the form ${ }^{10}$

$J(\overrightarrow{\mathrm{q}})$

$$
=\sum_{n, n^{\prime}, \overrightarrow{\mathrm{k}}} \frac{I_{n n^{\prime}}(\overrightarrow{\mathrm{k}}, \overrightarrow{\mathrm{k}}+\overrightarrow{\mathrm{q}}) f\left(\epsilon_{n}(\overrightarrow{\mathrm{k}})\right)\left[1-f\left(\epsilon_{n^{\prime}}(\overrightarrow{\mathrm{k}}+\overrightarrow{\mathrm{q}})\right)\right]}{\epsilon_{n^{\prime}}(\overrightarrow{\mathrm{k}}+\overrightarrow{\mathrm{q}})-\epsilon_{n}(\overrightarrow{\mathrm{k}})},
$$

where $\epsilon_{n}(\vec{k})$ is the energy of a Bloch state of wave vector $\overrightarrow{\mathrm{k}}$ in band $n, I_{n n^{\prime}}(\overrightarrow{\mathrm{k}}, \overrightarrow{\mathrm{k}}+\overrightarrow{\mathrm{q}})$ is a slowly varying function, and $f(\epsilon)$ is the Fermi function.

The peaks in $J(\vec{q})$ probably reflect the contribution of a large number of small energy denominators at $\vec{q}$ values corresponding to the separation between the approximately parallel electron and hole sheets of the Fermi surface. These are believed to be of crucial importance in determing $\vec{Q}$ in the heavy rareearth metals. ${ }^{11}$ Measurements at intermediate temperatures show that these peaks are abruptly flattened at the ferromagnetic transition, and this may be ascribed to the splitting of the different spin bands by the ferromagnetic exchange interaction.

We have studied the magnon lifetimes in the ferromagnetic phase by measuring the neutron group widths. The widths for the alloy, shown in Fig. 2(b), are within the experimental error the same as those for pure $\mathrm{Tb}$, where the latter have been measured at small and large $\vec{q}$. Since these widths are furthermore not observably temperature dependent, the principal mechanism limiting the magnon lifetime is probably absorption by the conduction electrons. ${ }^{12}$ This process involves a spin flip by the electron and the absorption may therefore decrease rapidly at critical wave vectors determined by the separation of the Fermi surfaces of different spin. For the free-electron model, the width well above the critical $\vec{q}$ is given $b^{12}$

$$
\Delta E(\overrightarrow{\mathrm{q}})=c E(\overrightarrow{\mathrm{q}}) / q,
$$

where $E(\vec{q})$ is the magnon energy and $c$ is a constant. This function, fitted at $q=0.72 \AA^{-1}$, is also shown in Fig. 2(b). The widths fall rather abruptly at about $q=0.35 \AA^{-1}$ and this may reflect the exchange splitting of the Fermi surface. The residual broadening at low 

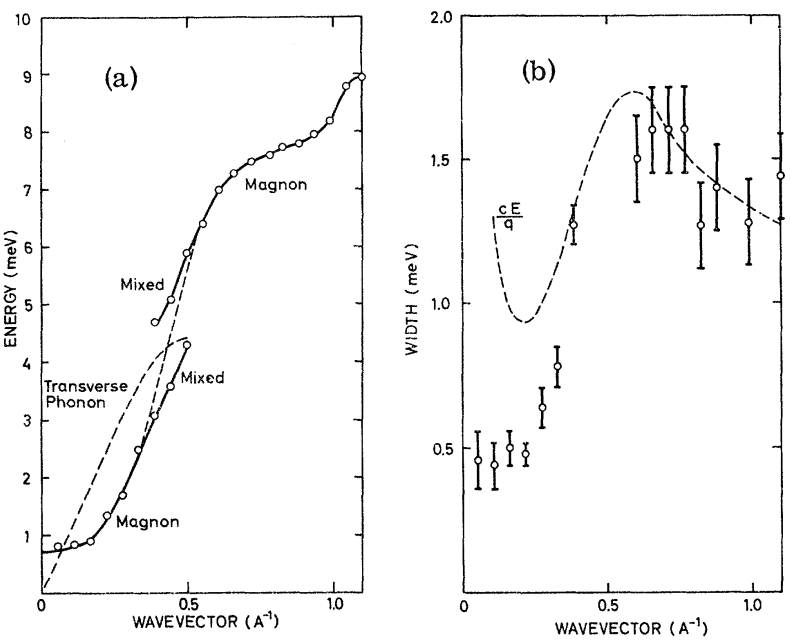

FIG. 2. (a) The dispersion relation for excitations in the $c$ direction in $\mathrm{Tb}-10 \% \mathrm{Ho}$ at $110^{\circ} \mathrm{K}$. The scan used for these measurements was such that transverse phonons were not observed. (b) Natural width of magnons propagating in the $c$ direction at $110^{\circ} \mathrm{K}$. The experimental resolution, which was always smaller than the natural width, has been extracted from the measurements.

$q$ is probably due to Umklapp processes of the type discussed by Luther and Tanaka. ${ }^{13}$

Because of their smaller spin, the Ho moments in the alloy are coupled comparatively weakly to the $\mathrm{Tb}$ host moments, and this produces a resonant-magnon mode. ${ }^{14}$ We have observed such a mode, manifested by a rapid change of energy with wave vector, relative to that in pure $\mathrm{Tb}$, which has the characteristic form associated with a resonant mode. At $110^{\circ} \mathrm{K}$, it occurs at $4 \mathrm{meV}$ in the acoustical magnon branch in the $a$ direction. The excitation energies in the $c$ direction at this temperature are shown in Fig. 2(a). At the crossing of the magnon and transverse-phonon dispersion curves, there is a strong interaction, leading to the creation of mixed magnon-phonon modes. The splitting at the crossing point of the unperturbed dispersion curves is 1.7
$\mathrm{meV}$, which is approximately the same magnitude as has previously been observed in a magnetic insulator. ${ }^{15}$ The magnon-phonon interaction is much weaker in pure $\mathrm{Tb}$, and no coupling has been observed in the alloy between magnons and transverse phonons propagating in the $a$ direction, for which the crossing occurs at much higher energy, It seems possible, therefore, that the coupling is enhanced by the large spin and charge deviations near the Ho impurities at the resonance frequency which, fortuitously, is close to the crossing of the magnon and phonon dispersion curves.

We have benefited greatly from discussions with Dr. R. J. Elliott, Professor J. R. Schrieffer, Dr. M. F. Collins, Dr. W. C. Marshall, and Dr. M. Blume.

\footnotetext{
${ }^{1}$ K. Niira, Phys. Rev. 117, 129 (1960).

${ }^{2}$ K. Yosida and H. Miwa, J. Appl. Phys. $32,8 \mathrm{~S}$ (1961).

${ }^{3}$ H. Bjerrum Møller and J. C. Gylden Houmann, Phys. Rev. Letters 16, 737 (1966).

${ }^{4}$ W. Brinkman, J. Appl. Phys. 38, 939 (1967);

W. Brinkman and R. J. Elliott, to be published.

${ }^{5} \mathrm{H}$. Bjerrum Møller and J. C. Gylden Houmann, Rare Earth Conference, Durham, North Carolina, 1966 (to be published).

${ }^{6}$ S. V. Tyablikov, Ukr. Mat. Zh. 11, 287 (1959); see also H. B. Callen, Phys. Rev. 130, 890 (1963).

${ }^{7}$ P. A. Lindgård, A. Kowalska, and P. Laut, to be published.

${ }^{8}$ J. J. Rhyne and A. E. Clark, J. Appl. Phys. $\underline{38}$, 1379 (1967).

${ }^{9} \mathrm{~J}$. R. Schrieffer, private communication.

${ }^{10}$ L. M. Roth, H. J. Zeiger, and T. A. Kaplan, Phys. Rev. 149,519 (1966).

${ }^{11}$ R. W. Williams, T. L. Loucks, and A. R. Mackintosh, Phys. Rev. Letters $\underline{16}, 168$ (1966).

${ }^{12}$ R. J. Elliott and H. Stern, Inelastic Scattering of Neutrons in Solids and Liquids (International Atomic Energy Agency, Vienna, 1961).

${ }^{13} \mathrm{~A}$. H. Luther and T. Tanaka, to be published.

${ }^{14}$ T. Wolfram and J. Callaway, Phys. Rev. 130, 2207 (1963).

${ }^{15}$ G. Dolling and R. A. Cowley, Phys. Rev. Letters $\underline{16}$, 683 (1966).
} 